\title{
NUEVA ESPECIE DE PASSALUS FABRICIUS, 1792 (COLEOPTERA: SCARABAEOIDEA: PASSALIDAE) DE LA SIERRA NEVADA DE SANTA MARTA, COLOMBIA
}

\author{
Larry JiMÉNEZ-FERBANS, ${ }^{1}$ Germán AMAT-GARCÍA ${ }^{2}$ \& Pedro \\ REYES-CASTILLO ${ }^{1}$ \\ ${ }^{1}$ Instituto de Ecología, A.C., Red de Biodiversidad y Sistemática. Carretera antigua a Coatepec 351, El \\ Haya, Xalapa 91070, Veracruz, México.<larryjimenezferbans@gmail.com>; <pedro.reyes@inecol. \\ edu.mx> \\ ${ }^{2}$ Instituto de Ciencias Naturales, Universidad Nacional de Colombia. Bogotá D. C., Colombia. \\ gdamatg@unal.edu.co
}

Jiménez-Ferbans, L., Amat-García, G. \& Reyes-Castillo, P. 2012. Nueva especie de Passalus Fabricius, 1792 (Coleoptera: Scarabaeoidea: Passalidae) de la Sierra Nevada de Santa Marta, Colombia. Acta Zoológica Mexicana (n. s.), 28(3): 607-612.

RESUMEN. Se describe e ilustra Passalus (Pertinax) unimagdalenae sp. n., especie de montaña distribuida entre los 1560 y 2309 msnm en la Sierra Nevada de Santa Marta.

Palabras clave: Taxonomía, Passalini, subgénero Pertinax, pasálidos, Ceroxylum ceriferum.

Jiménez-Ferbans, L., Amat-García, G. \& Reyes-Castillo, P. 2012. A new species of Passalus Fabricius, 1792 (Coleoptera: Scarabaeoidea: Passalidae) from Sierra Nevada de Santa Marta, Colombia. Acta Zoológica Mexicana (n. s.), 28(3): 607-612.

ABSTRACT. Passalus (Pertinax) unimagdalenae sp. n. is described and illustrated as a mountain species distributed between 1560 and 2309 masl in the Sierra Nevada de Santa Marta.

Key words: Taxonomy, Passalini, Pertinax subgenus, bess beetles, Ceroxylum ceriferum.

\section{INTRODUCCIÓN}

Con más de 140 especies, Passalus constituye el mayor género de Passalidae del Nuevo Mundo (Reyes-Castillo 2002), dominando la composición de la pasalidofauna de Sudamérica, tanto en tierras bajas como en montañas. En Colombia el género concentra el $63 \%$ de las especies de la familia; la mayoría representan elementos de amplia distribución en la región amazónica y los endemismos están concentrados en las zonas medias y altas de las cordilleras andinas.

Recibido: 21/09/2012; aceptado: 16/10/2012. 
Tradicionalmente, Passalus ha sido dividido en los subgéneros Mitrorhinus, Pertinax y Passalus s. str.; si bien los límites entre estos no son claros, por lo que Fonseca (1987) y Boucher (2006) sugieren la polifilia del género. Passalus (Mitrorhinus) está constituido por un pequeño grupo de 10 especies; en tanto que $P$. (Pertinax) y Passalus s. str. agrupan a la mayoría de las entidades. Como lo muestran Jiménez-Ferbans et al. (2010), en Colombia Passalus s. str. tiende a dominar la composición en las tierras bajas; en tanto que, Pertinax muestra una mayor riqueza en altitudes medias. Jiménez-Ferbans et al. (2010) presentan los resultados de muestreos realizados a lo largo de un gradiente altitudinal en la Sierra Nevada de Santa Marta y citan numerosos ejemplares determinados como Passalus (Pertinax) sp. n., en virtud de su antena trilamelada, lacinia bidentada y borde frontal anterior sin dientes secundarios. Recientemente estudiamos ese material y concluimos que se trata de una nueva especie, cuya descripción se da a conocer en la presente contribución.

\section{MATERIALES Y MÉTODOS}

Se examinaron 58 ejemplares depositados en las colecciones entomológicas siguientes: Instituto de Ciencias Naturales (ICN) de la Universidad Nacional de Colombia; Universidad del Magdalena (CEUM), Colombia; Instituto de Ecología (IEXA), México; y Universidad del Valle de Guatemala (UVGC), Guatemala. Los ejemplares fueron comparados con especies representativas de distintos grupos dentro de $P$. (Pertinax); dado que los especímenes presentan afinidad con $P$. punctatostriatus Percheron, 1835, se contrastaron las descripciones originales de los sinónimos para esta especie listados por Hincks \& Dibb (1935). La descripción se basa en la terminología propuesta por Reyes-Castillo (1970) y los dibujos se realizaron a la cámara clara.

\section{RESULTADOS TAXONÓMICOS}

Passalus (Pertinax) unimagdalenae sp. $\mathrm{n}$.

HOLOTIPO $\overbrace{}^{\top}$ : COLOMBIA: Magdalena, Sierra Nevada de Santa Marta, San Lo-

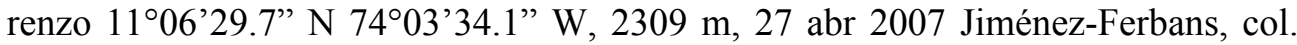

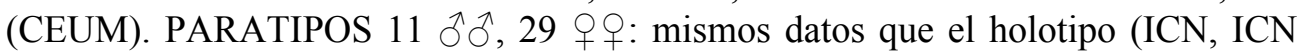
007777, UVGC, IEXA). PARATIPOS 1 đึ y 7 $q$ : COLOMBIA: Magdalena, Sierra Nevada de Santa Marta, ver. Bella Vista, $11^{\circ} 05^{\prime} 46^{\prime \prime}$ N 7404' 51.7” W, 1560 m, 28 abr 2007, Jiménez-Ferbans, col. (ICN, CEUM). PARATIPOS 4 đ̋ $\overbrace{}^{\Uparrow}$ y 5 우우: COLOMBIA: Cesar, Valledupar, Sierra Nevada de Santa Marta, corr. Guatapurí, $10^{\circ} 44^{\prime} 00^{\prime}$ ' N 7324' 6" W, 1795 m, 05 jul 07, Jiménez-Ferbans \& M. Mejía, cols. (ICN, ICN 00679, ICN 00680, ICN 007715, ICN 007716, ICN 007717, CEUM).

Diagnosis. Passalus unimagdalenae sp. n. es similar a $P$. punctatostriatus, del que se diferencia fácilmente por su menor tamaño, tubérculos internos grandes y de ápice 

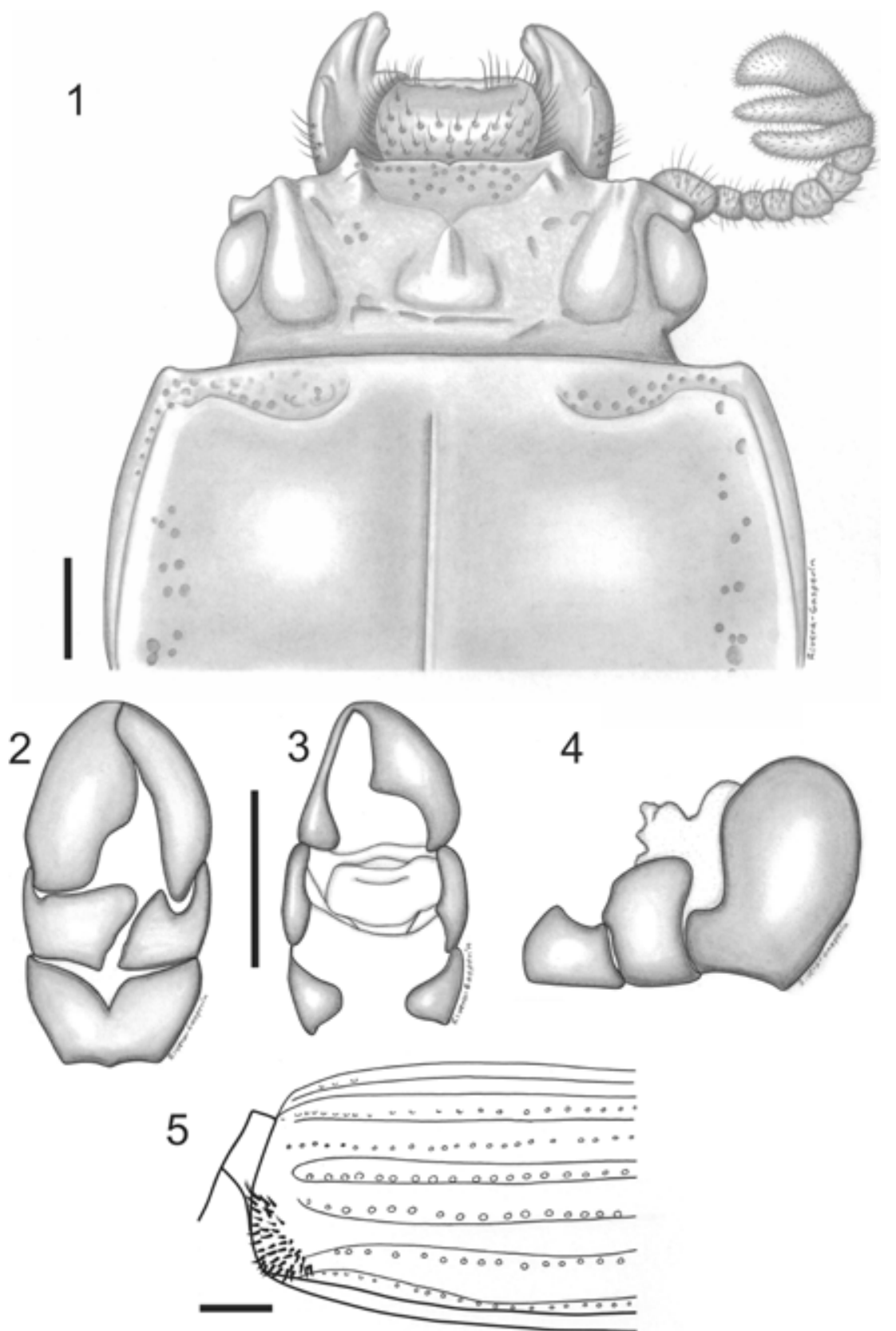

Figuras 1-5. 1: Cabeza y parte anterior del pronoto de Passalus unimagdalenae n. sp., vista dorsal. 2: Edeago vista ventral. 3: Edeago vista dorsal. 4: Edeago vista lateral. 5: Parte anterior del élitro, vista lateral. 
libre, humeri con sedas largas en su base y parámeros del edeago no fusionados entre sí, ni con la pieza basal.

Descripción. Habitus: talla pequeña, 20.3-22.9 mm de largo total, macróptero, cuerpo aplanado de color negro brillante.

Cabeza: labro con borde anterior recto, cubierto por sedas que se hacen menos densas en la parte anterior. Clípeo oculto debajo de la frente, con ángulos anteriores muy desarrollados, de tamaño ligeramente mayor a los tubérculos externos. Borde frontal anterior con profunda hendidura media, sin tubérculos secundarios (Fig. 1). Tubérculos externos grandes, proyectados hacia adelante. Tubérculos internos de igual tamaño que externos y próximos a la base de éstos, de ápice libre. Tubérculos externos e internos no unidos por quilla. Los tubérculos internos se unen a la estructura media frontal por quillas en forma de "V". Área entre las quillas frontales con puntos que pueden ser escasos o abundantes (5-24 puntos), y marcado mamelón justo en la base de las quillas. Estructura media frontal tipo falsus (Reyes-Castillo 1970), con quilla central pequeña, de ápice no libre y tubérculos laterales marcados. Fosas frontales glabras, sin puntos o sólo unos cuantos en las proximidades de los tubérculos internos. Ojos grandes, el canthus ocular ocupa 1/4 del ancho del ojo en vista lateral. Surco occipital marcado y completo, de forma semicircular. Surco posocular no definido. Gula arqueada en borde anterior. Proceso hipostomal ligeramente separado del mentón, glabro y largo, alcanzando la parte superior de la zona media del mentón. Parte media basal del mentón ventralmente sobresaliente y glabra. Maza antenal trilamelada, con artejos cortos.

Mandíbula tridentada en el ápice, con dientes de casi el mismo tamaño. Diente interno bífido en la mandíbula izquierda y monocolumnar en la derecha. Diente dorsal recto en vista dorsal y plano en vista lateral. La pubescencia dorsal de las mandíbulas alcanza toda la base del diente móvil. Foseta mandibular larga, cubriendo toda la base del diente móvil. Maxilas con lacinia bidentada en el ápice. Lígula tridentada, con diente medio de largo mayor que los laterales. Artejo medio del palpo labial de igual ancho y ligeramente menos largo que el artejo distal.

Tórax: Pronoto con punteado lateral abundante, concentrado en las proximidades de las fosetas laterales. Ángulos anteriores ligeramente agudos. Surco marginal ocupando más de la mitad del margen anterior del pronoto. Surco longitudinal bien definido. Foseta lateral marcada. Prosternelo romboidal truncado. Brazos pronotales brillantes y pubescentes. Mesosternón glabro, salvo por dos grupos de sedas cortas en su margen anterior, cubiertos por el prosternón. Cicatriz mesosternal alargada y marcada. Esquina posterior del mesepisternón y mesepímero glabros. Disco metasternal liso, delimitado por pocos puntos (20-30) en su parte media y posterior. Parte anterolateral del metasternón glabra; foseta lateral glabra, de menor ancho que la tibia II.

Élitros: brillantes, borde anterior rectangular y glabro. Humeri con sedas largas en su base, epipleura glabra (Fig. 5). Estrías laterales con puntuación redonda marcada. 
Patas: profémur con surco marginal anterior marcado y ancho, alcanzando la pubescencia apical. Tibia I con surco dorsal incompleto. Tibias II y III desarmadas o con una o dos espinitas débiles.

Abdomen: Surco marginal del último esternito completo.

Edeago: vista ventral (Fig. 2): pieza basal separada de los parámeros, con leve hendidura en zona media. Parámeros no fusionados entre sí. Lóbulo medio alargado, ocupando $1 / 2$ del largo total del edeago, medido en la línea media ventral.Vista lateral: proyección lateral de los parámeros corta y redondeada. Parámero izquierdo con prominente proyección ventral (Fig. 4).

Variación. Los ejemplares de San Lorenzo, incluyendo el holotipo, son de mayor talla que los de menor altitud; por otra parte, algunos ejemplares pueden tener el área frontal casi totalmente cubierta de puntos, en tanto que otros sólo presentan tres puntos aislados.

Etimología. Dedicamos esta especie en homenaje a la Universidad del Magdalena, al cumplirse 50 años de su fundación, durante los cuales ha contribuido a la investigación biológica en el Caribe colombiano.

Ecología. Especie común en la Sierra Nevada de Santa Marta, donde sobre la vertiente noroccidental vive en troncos caídos de árboles del bosque muy húmedo premontano situado a 1560 msnm (Espinal \& Montenegro 1963), y en el bosque muy húmedo montano bajo ubicado a $2309 \mathrm{~m}$ de altitud, ha sido recolectado en ramas caídas de árboles y en un tallo en descomposición de la "palma de cera" Ceroxylum ceriferum (H. Karst) Pittier (Arecaceae) se recolectaron adultos y larvas. En la vertiente Suroccidental de esta Sierra, se encuentra en troncos podridos de árboles del bosque húmedo premontano situado a $1795 \mathrm{msnm}$. Estos tres tipos de bosque húmedo presentan pluviosidad anual entre 2546 y $2641 \mathrm{~mm}$.

\section{DISCUSIÓN}

Passalus (Pertinax) unimagdalenae sp. n. está restringida a la zona de altitud media (1560-2309 msnm) de la Sierra Nevada de Santa Marta. Jiménez-Ferbans et al. (2010) la citan como la única especie colectada por arriba de los $2000 \mathrm{msnm}$ y en muestreos realizados posteriormente por los autores, se encontró de forma abundante en la zona de San Lorenzo (2309 msnm).

En el bosque muy húmedo montano de San Lorenzo las palmas son importantes por su abundancia y frecuencia, tanto por el número de especies como de individuos. Ceroxylum ceriferum crece en los bosques húmedos de la vertiente norte de la Sierra Nevada de Santa Marta (La Guajira y Magdalena) y en la Serranía de Perijá (Cesar y La Guajira) entre 1900 y $2800 \mathrm{msnm}$. Sus poblaciones tienen una distribución restringida, lo que acompañado de la disminución en la calidad del hábitat colocan esta especie en peligro de extinción (Galeano \& Bernal 2010). 
La mayoría de los pasálidos registrados en palmeras podridas se han encontrado en número reducido y casi siempre en estado adulto, rara vez en grupos familiares con sus estados de desarrollo; en general se trata de especies de amplia distribución, abundantes y frecuentes en troncos de dicotiledóneas. Castillo \& Reyes-Castillo (2003) reseñan 14 especies de Passalidae registrados en troncos podridos de palmeras en el neotrópico, regiones etiópica y australiana.

Resultan evidentes las afinidades de $P$. unimagdalenae con $P$. punctatostriatus, una especie distribuida desde México hasta Brasil (Hincks \& Dibb 1935). Sin duda, la amplia distribución de $P$. punctatostriatus es consecuencia de la inclusión como sinónimos de entidades que en realidad son diferentes. Por ello, se hace necesaria una revisión exhaustiva de los tipos de los sinónimos incluidos por Hincks \& Dibb (1935) dentro de esta especie, a fin de esclarecer su estatus taxonómico.

Agradecimientos. Agradecemos a Margarita Mejía por su colaboración en la recolecta de la serie tipo y a Sara Rivera por la elaboración de las ilustraciones. Esta publicación es parte del trabajo doctoral de Larry Jiménez-Ferbans, que es financiado por el CONACYT-México mediante el proyecto 169604.

\section{LITERATURA CITADA}

Boucher, S. 2006. Évolution et phylogénie des coléoptères Passalidae (Scarabaeoidea). Les taxons du groupe famille. La tribu néotropicale des Proculini et son complexe Veturius. Annales de la Société Entomologique de France, 41: 239-603.

Castillo, M. L. \& Reyes-Castillo, P. 2003. Los Passalidae: coleópteros tropicales degradadores de troncos de árboles muertos, pp. 237-262. In: J. Álvarez-Sánchez \& E. Naranjo García (Eds.). Ecología del suelo en la selva tropical húmeda de México. Instituto de Ecología, A.C., Instituto de Biología y Facultad de Ciencias, UNAM. Xalapa, México.

Espinal, L. S. \& Montenegro, E. 1963. Formaciones vegetales de Colombia. Instituto Geográfico Agustín Codazzi, Bogotá, Colombia.

Fonseca, C. R. V. 1987. Sistemática filogenética e biogeografía dos Passalidae (Coleoptera) do mundo. Tese Doctorado-Zoologia do Instituto de Biociencias da Universidade de Sao Paulo. Brasil. 158 pp.

Galeano, G. \& Bernal, R. 2010. Palmas de Colombia. Guía de Campo. Editorial Universidad Nacional de Colombia. Instituto de Ciencias Naturales, Facultad de Ciencias-Universidad Nacional de Colombia, Bogotá. 688 pp.

Hincks, W. D. \& Dibb, J. R. 1935. Passalidae. Coleopterorum Catalogus, pars 142. W. Junk's-Gravenhage, $118 \mathrm{pp}$.

Jiménez-Ferbans, L., Amat-García, G. \& Reyes-Castillo, P. 2010. Diversity and distribution patterns of Passalidae (Coleoptera Scarabaeoidea) in the Caribbean Region of Colombia. Tropical Zoology, 23: 147-164.

Reyes-Castillo, P. 1970. Coleoptera, Passalidae: morfología y división en grandes grupos; géneros americanos. Folia Entomológica Mexicana, 20-22: 1-217.

Reyes-Castillo, P. 2002. Passalidae, pp. 467-483. In: J. Llorente Bousquets \& J. J. Morrone (Eds.). Biodiversidad, taxonomía y biogeografía de artrópodos de México: hacia una síntesis de su conocimiento. Volumen III. Universidad Nacional Autónoma de México, México, D. F. 